\title{
Simulation Design of Semantic Diagnosis Based on Audio Sampling of Multi Modal Signal Control
}

\author{
Weilin Zou \\ Department of Chinese and Journalism, Changsha University, Changsha, 410003, China \\ weilin_zou@yeah.net
}

Keywords: Discrimination; Audio-visual expression; CFtool toolbox; Curve fitting; Multi-modal.

\begin{abstract}
The modern Chinese vocabulary is the inheritance of ancient Chinese vocabulary development. Although its development process has local changes, there are some similarities in the modal expression, and it can help to better grasp the analysis of ancient and modern meanings for the reasonable analysis. Accordingly, we use the multi modal identification algorithm in the discrimination of ancient and modern meanings, and analyze multi modal response from the audiovisual effect of the meaning. At the same time, using CF tool curve fitting toolbox carries on fitting for the common of ancient and modern meanings, we can get the results of modal response in image, sound and text, which provide a theoretical reference for the study of ancient and modern word sense discrimination.
\end{abstract}

\section{Introduction}

Some of the words in ancient times have died with the progress of the times, and some has been used till today. Some words of ancient and modern meanings not only have the change, and the difference is very large, including meaning extension, meaning acronym and meaning transfer [1]. But no matter how the change of ancient and modern meanings, the modal response mode can have some similarities, its commonality can be used to express the multi modal analysis, in which the data mining process is shown in Figure 1.

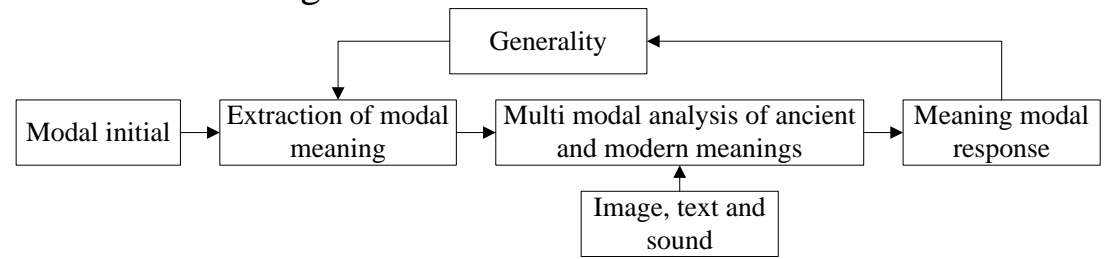

Fig.1: Schematic diagram of semantic multi-modal data mining process

Figure 1 shows the schematic diagram of semantic multi-modal data mining process. Firstly, we select the similar estimation words database and define initialization mode in the database, and then extract the meaning database to get the multi modal response model of the estimation of word meaning [2]. Secondly, combined with the analysis of image, text and sound effect, we can get the multi modal response curve fitting of estimated word meaning. Finally, we get the similarities and differences of ancient and modern meanings directly using the analysis of multi modal response curve fitting.

After a scattering of ultrasonic signal to is usually a broadband pulse in the center frequency modulation transient signal. Usually there is a time limit and frequency of the restrictions. The scattering noise usually can't be canceled. In the past often use average or band pass filter matching non-stationary signal analysis technology. And given the extensive attention. Based on this form of time and frequency analysis, wavelet transform, empirical mode decomposition. Through these methods, but the non-stationary signal characteristics of ultrasonic extraction of the useful components, so as to reduce the noise. Sparse decomposition provides an inherent signal structure, which can also be used to deal with ultrasonic nondestructive testing signals. Signal sparse decomposition, it is to point to by repeatedly onto a given a complete atomic library signal, and choose the most matching atomic library signal each iteration. Matching pursuit, is a greedy algorithm as the core to realize such a decomposition, and has been widely used in many signal 
processing field[3].

In general can use the signal frequency characteristics, to provide the signal of the sparse information, also can be used in ultrasonic nondestructive signal processing. Based on the signal of the principle of sparse solution of the new type ultrasonic nondestructive signal processing algorithm. In order to reduce the noise, with matching pursuit of the algorithm for the selected signal decomposition, can in the location of the echo quantitative information analysis and prediction, such as the size and location of the crack. This research is to use computer simulation signal and experimental ultrasonic algorithm, from more than a thin metal plate of artificial hole to measure back scatter echo signal.

In this paper, the matching pursuit algorithm, and on the basis of the decomposition of the function of our Gabor of ultrasonic signal. By inhibiting noise to improve flaw and use of information signal sparse to estimate the size and location of the crack the algorithm used in ultrasonic testing and simulation signal, the experimental results show that it in strong noise to signal the presence of the treatment did well[4]. In addition, the proposed method conform to the requirements of the quantitative condition. Based on the matching of the tracking Gabor function, and, to some extent, using ultrasonic nondestructive signal processing can because it to evaluate the cracks of the size.

\section{Meaning Multimodal Analysis Algorithm Theory}

In the process of meaning multimodal analysis, we require a combination of ancient and modern meanings. Combined with semantic information data in the analysis model of the lexical meaning, hypothesis lexical multimodal collection has $r$ meaning tag, and each tag defines a weight that is $D_{i}$, so the multi modal meaning expression is shown in formula (1).

$$
w(t, p)=\frac{T F(t, p) \times \lg \left(M / m_{k}+0.01\right)}{\sqrt{\sum_{t \in d}\left[T F(t, p) \times \lg \left(M / m_{k}+0.01\right)\right]^{2}}}\left[1+b(t, p) \times \frac{\sum_{l \in L} D_{l}}{\sum_{i=1}^{r} D_{i}}\right] .
$$

Where $b(t, p)$ represents the meaning of a word, when $t$ is in multi mode, its value is 1 ; when $t$ is outside the time, its value is $0 . L$ represents language multimodal tag set. Hypothesis that $k$ represents the initial mode, and $j$ represents the updated modal, then modal weights are expressed as follows:

$$
S(p)=(k-j+1) / k .
$$

So, we can add semantic information data in the mode, as shown in the formula (3).

$$
A w(p)=\sum_{m=1}^{k} k(p, m) / k
$$

The whole weight of lexical information filtering can be expressed as

$$
\operatorname{Iw}(p)=S(p)+A w(p)
$$

After the expression multi-modal response model of multiple meanings is modified, we can eventually obtain the modal expression of the estimation of word discrimination.

$$
w(t, p)=\frac{T F(t, p) \times \lg \left(M / m_{k}+0.01\right)}{\sqrt{\sum_{t \in d}\left[T F(t, p) \times \lg \left(M / m_{k}+0.01\right)\right]^{2}}} \times\left[1+b(t, p) \times \frac{\sum_{l \in L} D_{l}}{\sum_{i=1}^{r} D_{i}}+I w(p)\right] .
$$

The matching pursuit algorithm is a kind of practical algorithm, is from a complete library, select a signal, and making linear decomposition, form a expansion of the waveform signal. For other signal optimization technique for, matching pursuit algorithm is a greedy algorithm, in place some place to find not ideal, and its basic principles can be described as follows:

$$
f=\sum_{n=0}^{m-1}\left(R^{n} f, g_{r_{n}}\right) g_{r_{n}}+R^{m} f
$$




$$
\|f\|^{2}=\sum_{n=0}^{m-1}\left|\left\langle R^{n} f, g_{r_{n}}\right\rangle\right|^{2}+\left\|R^{m} f\right\|^{2}
$$

Iteration of the surplus, (for example), the best matching of the residue of the surplus. We define and belong to, we assume that meet the:

$$
\left|\left\langle R^{n} f, g_{r_{n}}\right\rangle\right| \geq a, \sup _{\gamma \in \tau}\left|\left\langle R^{n} f, g_{r_{n}}\right\rangle\right|, \quad 0<a \leq 1
$$

Matching pursuit algorithm is the last remnants of the study of optimal matching, after such a many iterative process, The surplus energy in limited dimension of space where the form of exponential decay[5].

According to above mathematical model, using CFtool fitting toolbox carries out programming for the algorithm, in which the used main program are as follows [6]:

Linear model Poly3:

$$
f(x)=a{ }^{*} x^{\wedge} 3+a 2 * x^{\wedge} 2+a 3 * x+a 4
$$

Coefficients (with 95\% confidence bounds):

$$
\begin{aligned}
& \text { a1 }=9(9,9) \\
& \text { a2 }=8(8,8) \\
& \text { a3 }=4.593 \mathrm{e}-15(-3.266 \mathrm{e}-14,4.185 \mathrm{e}-14) \\
& \text { a4 }=1(1,1)
\end{aligned}
$$

Goodness of fit:

SSE: 2.386e-28

R-square: 1

Adjusted R-square: 1

RMSE: 5.839e-15

\section{The Multimodal Analysis of Ancient and Modern Word Discrimination Based on CFtool} Fitting Tool

In order to verify the effectiveness and reliability of multimodal analysis algorithm designed in the second part, this paper analyzes the multimodal response of ancient and modern meanings using CF tool curve fitting toolbox, including the use of CFtool toolbox interface as shown in Figure 2.

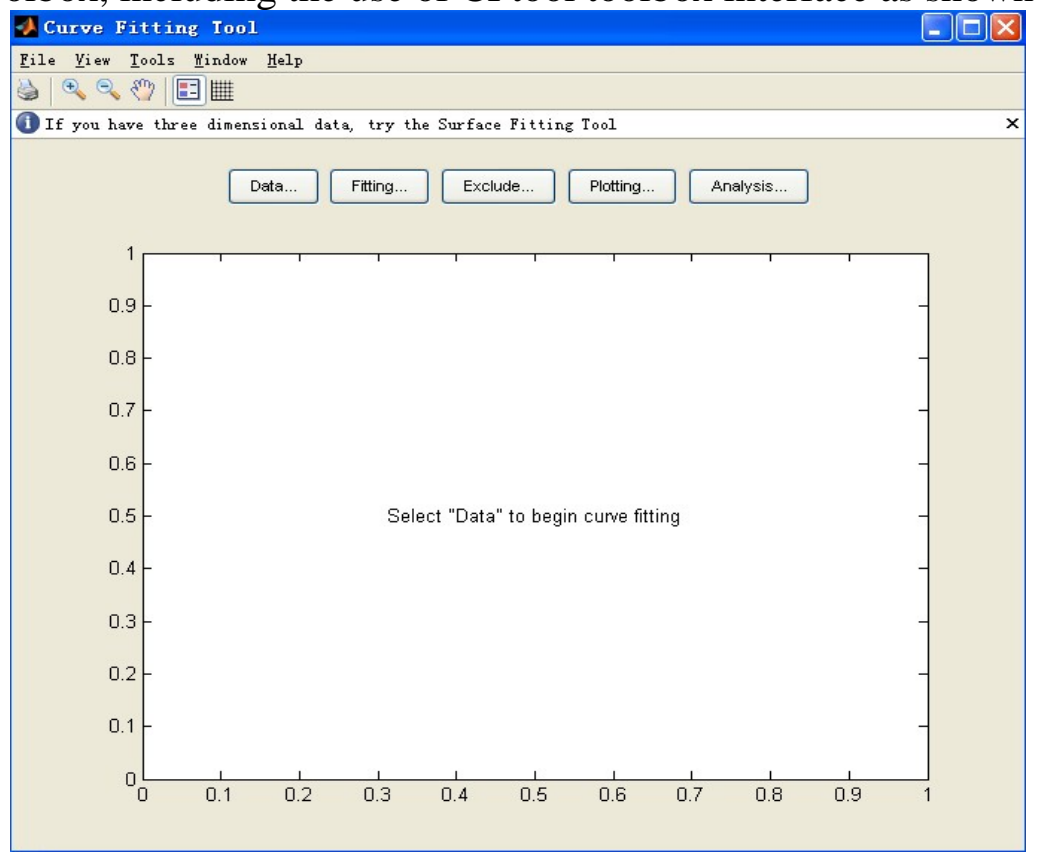

Fig.2: CFtool curve fitting toolbox 
Figure 2 shows the CFtool curve fitting toolbox of MATLAB, the tool has simple and practical and powerful curve fitting function, which provides a rich fitting algorithm to achieve a variety of linear and nonlinear curve fitting [7,8]. First, the language of ancient and modern meanings inputs into the toolbox as shown in Figure 3.

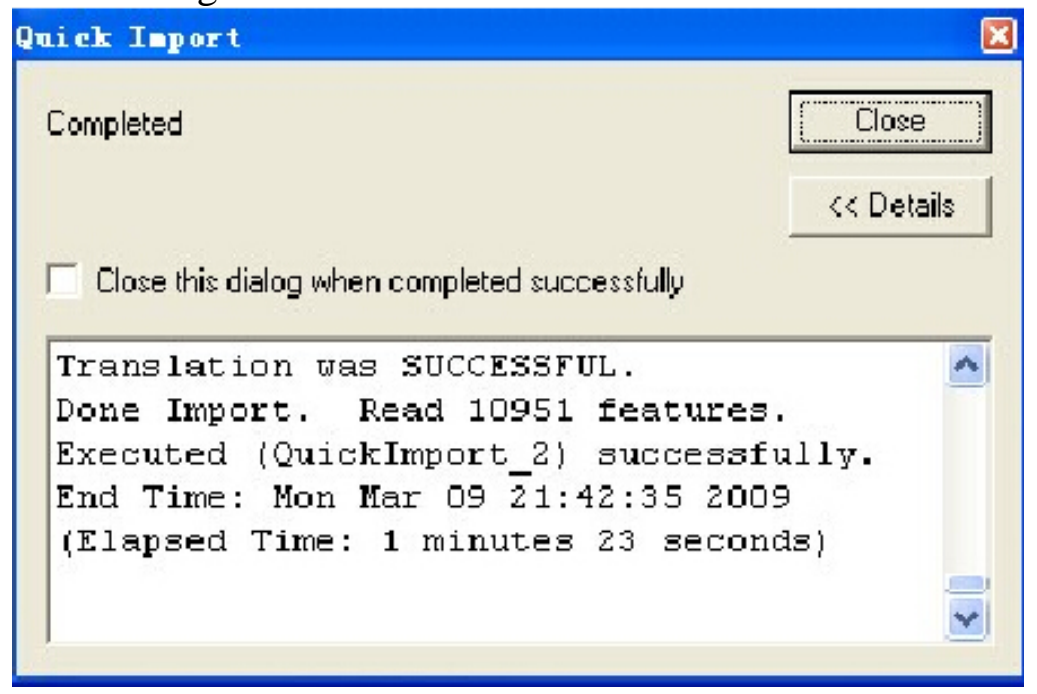

Fig.3: Schematic diagram of data conversion

Figure 3 shows the data import process of ancient and modern meaning multi modal analysis. Firstly, in the network using the Web searches engine the similar meaning in ancient and modern literature [9].Secondly, we search the modal response mode in the meaning expression multi modal, and then we analyze the characteristic of voice, image and text expression symbols of ancient and modern meanings from the visual, auditory and tactile [10].Finally, symbol features will be imported into the software to realize the data conversion process.

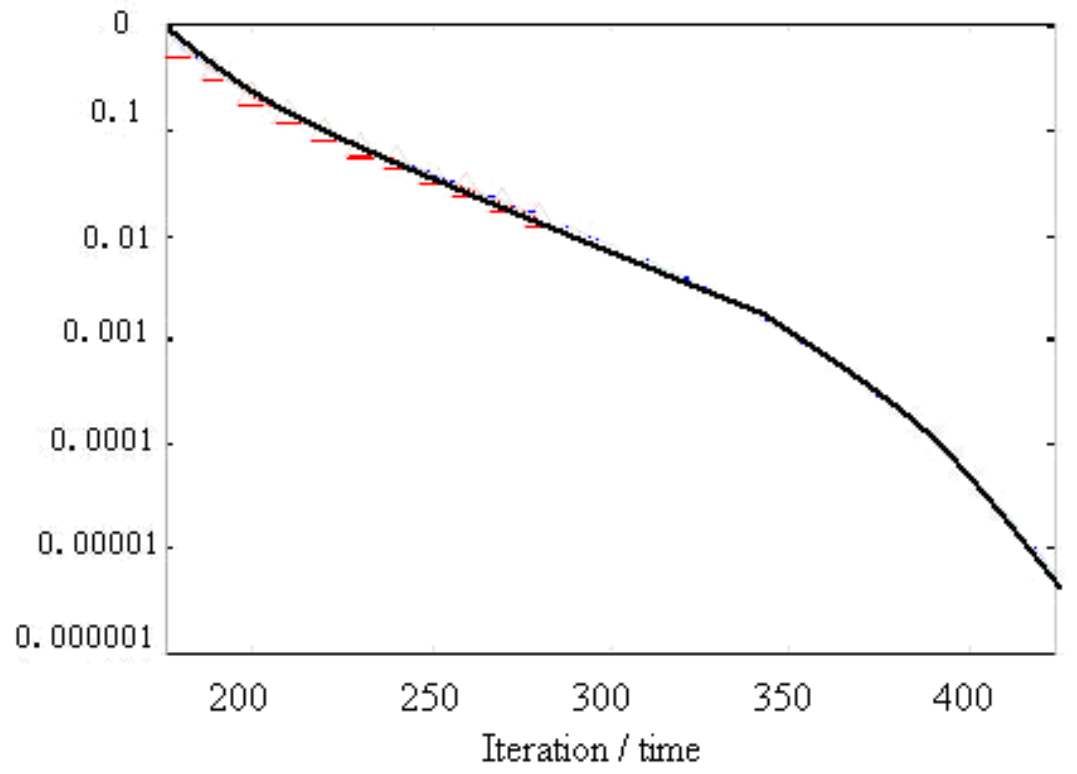

Fig.4: The fitting curve of ancient and modern word discrimination

Figure 4 shows the fitting curve of ancient and modern word discrimination are drawn by MATLAB curve fitting toolbox [11]. It can be seen from the chart that with the iteration number increases, the ancient and modern meaning has a very good fit in the multi modal analysis of the word discrimination, in which the common performance is the multi modal characteristics of visual, auditory and tactile. 


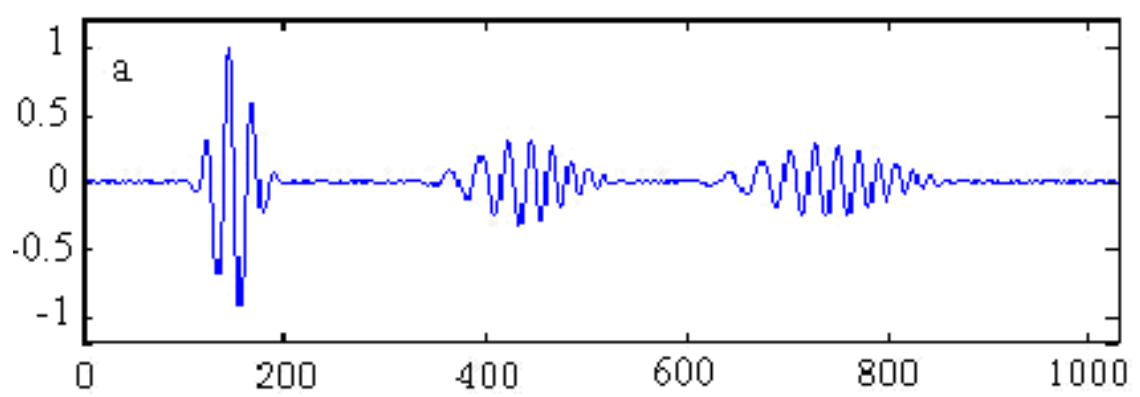

Fig.5: The multi modal response curve of ancient and modern word discrimination

Figure 5 shows the response curve of ancient and modern meanings in multi modal analysis, it can be seen from the chart that in the meaning of the expression, ancient and modern meanings in the three modes has obvious modal response, the three modes are respectively visual, auditory and tactile, and corresponding modal are respectively images, sound and text, in order to verify the estimation of meaning in multi modal analysis, this shows that although the estimated meaning has different expression ways, the expression form and mode have good fusion.

From Figure6, it is known that the model that proposed in this paper is correct. The reason is that the thesis adopted Verilog-AMS method to have the modeling, the model had the top-down method to be established, and it didn' t refer to the specific circuit. Because this paper reasonably used the Verilog-AMS method to have modeling, this made the mixed signal integrated circuit model reasonable.

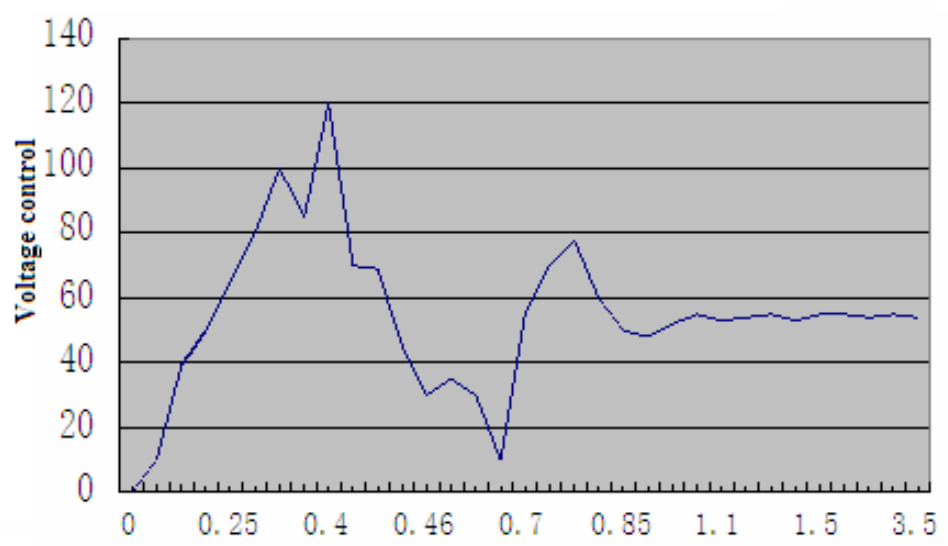

Fig.6: Simulation diagram of the change of voltage control

Industrial users should take into account that application programs apply to the monitoring and control of the LR-WPAN such as technology type. Its reliability, security and performance, the design of wireless technology, deployment and such factors e exist potential challenges, such as EMI / RFI multipath fading in industrial environments are specific issues can be considered. The provided appropriate network topology; it has overcome all of the problems caused due to the industry environment. In a variety of environmental conditions, we tested applications of a network used star network with four nodes in the industrial, such as speed and direction control of DC motors, lighting control of incandescent lamp and closed control of the temperature of the circulating water path. In the future, we can also test other suitable network of wireless data communication, in order to achieve the extensive application of wireless safety communications, realize the effective conduct of industrial automation and control, promote economic development and better create benefits for society.

\section{Summary}

(1) On the basis of multimodal recognition theory, combining with the common interpretation of modern meaning and ancient meaning, we have conducted the thorough research on the multi modal response characteristics of ancient and modern meanings, to obtain the multi modal response mathematic model and algorithm of the estimation word meaning. At the same time, the use of 
numerical simulation of CFtool curve fitting toolbox calculation can obtained multi modal response fitting curve of the estimation meaning.

(2) Through the modal analysis of ancient and modern meanings in image, sound and text, we obtain the visual, auditory and tactile mode common, so we need to further study on more mode common, which provide an the more favorable language environment for the understanding of ancient words.

\section{References}

[1] T. Liu, B. Qin, Y. Zhang. Search engine information retrieval practice. Beijing: China Machine Press, 2010:144-147.

[2] Q. Wang, H.W. Zhuang. Query suggestion based on TF-IQF model. Computer Engineering, 2010, 36(21): 78-80.

[3] Y.N. Li, S. Xu, B. Wang. Chinese query recommendation research based on weighted SimRank. Journal of Chinese information, 2010, 24(3): 4-9.

[4] C.G. Ma. Research on query expansion algorithm based on user interest. Harbin Engineering University, 2010:46-47.

[5] R. Chen. Realization and study of information retrieval query expansion model based on concept map. Northwestern University, 2011:19-24.

[6] W.J. Li, T.J. Zhao, X. Wang. Query expansion based on context. Journal of computer research and development, 2011, 47(2): 301-303.

[7] D.L. Zhang. Application of multimodal discourse theory and media technology in foreign language teaching. Foreign language teaching, 2012(4): 25-27.

[8] Q.H. Wei. Research on multi modal and college students' multiliteracies. Foreign language teaching research, 2011(2): 34-36.

[9] R. Gao, B.L. Zhao. Study of college English classroom teaching discourse. Border economy and culture, 2011(12): 102-104.

[10] D.L. Zhang, S.J. Zhang. Explore of multimodal foreign language textbooks compiling principle. Foreign language field, 2011(5): 56-58.

[11] C.Y. Yuan. Exploration on multi modal information cognition teaching and learning mode-the complex course "legal English" teaching reform. Shandong foreign language teaching, 2011(4): 120-123. 